\title{
New Solutions of Elastic Waves in an Elastic Rod under Finite Deformation
}

\author{
Peng Guo, ${ }^{1,2,3}$ Xiang $W u,,^{2,3}$ and Liangbi Wang ${ }^{2,3}$ \\ ${ }^{1}$ School of Mathematics and Physics, Lanzhou Jiaotong University, Lanzhou 730070, China \\ ${ }^{2}$ School of Mechatronic Engineering, Lanzhou Jiaotong University, Lanzhou 730070, China \\ ${ }^{3}$ Key Laboratory of Railway Vehicle Thermal Engineering, Lanzhou Jiaotong University, Ministry of Education, Lanzhou 730070, China \\ Correspondence should be addressed to Peng Guo; guopenglzjtu@126.com
}

Received 28 May 2014; Revised 19 August 2014; Accepted 19 August 2014; Published 1 September 2014

Academic Editor: Baolin Wang

Copyright (c) 2014 Peng Guo et al. This is an open access article distributed under the Creative Commons Attribution License, which permits unrestricted use, distribution, and reproduction in any medium, provided the original work is properly cited.

The nonlinear wave equation of an elastic rod under finite deformation is solved by the extended mapping method. Abundant new exact traveling wave solutions for this equation are obtained, which contain trigonometric function solutions, solitary wave solutions, Jacobian elliptic function solutions, and Weierstrass elliptic function solutions. The method can be used in further works to establish more entirely new solutions for other kinds of nonlinear evolution equations arising in physics.

\section{Introduction}

In the recent two decades, the studies of the nonlinear wave have made brilliant achievement. Many new and interesting phenomena have been discovered in various fields, such as physics and engineering. Solid mechanics in its application has scored great success in the studies of classical linear wave. In recent years, nonlinearities of solid structures have captured the attention of many researchers. More and more technological problems involve nonlinearity $[1,2]$. The nonlinear evolution equations (NLEEs) are widely used to describe complex phenomena in solid mechanics. It has aroused great interest in how the NLEEs can be qualitatively analyzed and solved.

In order to find the exact solutions, especially, traveling wave solutions of the NLEEs, various powerful methods are reported, such as inverse scattering method [3], Hirota bilinear method [4], the tanh method [5], the extended tanh method [6], sine-cosine method [7], homogeneous balance method [8], F-expansion method [9], the Exp-function expansion method [10], the mapping method [11], and the extended mapping method [12]. Among them, the mapping method is more convenient and effective than other analytic techniques.

The pioneer work of Lou and $\mathrm{Ni}$ [11] introduced the mapping method for a reliable treatment of the NLEEs.
The method is further developed and improved by many researchers such as [12] and by the references therein. Bai et al. [12] have successfully applied the extended mapping method to find solitary wave solutions, trigonometric function solutions, and Jacobian elliptic function solutions of several known NLEEs like Duffing equation, KleinGordon equation, sin-Gordon equation, Landau-GinzburgHiggs equation, and $\varphi^{4}$ equation.

In the present research, we are concerned with studying the nonlinear wave equation of an elastic rod under finite deformation, which was derived by Liu and Zhang [13] in 2006. They obtained solitary wave solutions for this equation by the hyperbolic secant function finite expansion method. Two kinds of nonlinear Schrödinger equations were derived from this equation by using the reductive perturbation method in [14]. Zhang and Liu [15] obtained solitary wave solutions and shock wave solutions for this equation by the Jacobi elliptic function expansion method. Finding new solution is still a worthwhile research to this equation. We use the extended mapping method to construct new solutions of the nonlinear wave equation of an elastic rod under finite deformation. We hope that this technique can be applied to find new solutions of other kinds of NLEEs.

This paper is organized as follows. In Section 2, the nonlinear wave equation of an elastic rod under finite deformation is derived by means of the Hamilton principle. 
The extended mapping method for finding exact traveling wave solutions of the NLEEs is described. In Section 3, the method to solve the nonlinear wave equation is illustrated in detail, and some novel solutions are discussed. Then, the conclusions are drawn in Section 4.

\section{The Governing Equation and the Extended Mapping Method}

Consider the nonlinear wave propagation in an infinite homogenous elastic straight circular rod with radius $R$. Use Lagrange material description and the cylindrical coordinate system as $(r, \theta, x)$, where $x$ is the axis of the rod, $r$ is radial, and $\theta$ is circumferential coordinate, respectively. To derive the governing equation, the following assumptions are introduced.

(i) Plane cross-section always remains plane in process of deformation.

(ii) The rod is subjected to axial symmetry compression, circumferential displacement $U_{\theta}=0$, and $\partial / \partial \theta=0$.

(iii) Due to the Poisson effect, the following relationship between the longitudinal displacement gradient $\partial U / \partial x$ and the radial displacement $U_{r}$ is valid; $U_{r}=-v r(\partial U / \partial x)$, where $U=U_{x}$ is the longitudinal displacement.

(iv) Consider the effect of the finite deformation and the axial strain-displacement relationship is given; that is, $\varepsilon_{x}=(\partial U / \partial x)+(1 / 2)(\partial U / \partial x)^{2}$. Because $U_{r}$ is higher order small quantity than $U$, the transverse shear strain is obtained: $\gamma=\left(\partial U_{r} / \partial x\right)=-v r\left(\partial^{2} U / \partial x^{2}\right)$.

(v) The relationship of stress and strain is linear; the strain energy density is quadratic function of strain.

Based on the above assumptions and Hamilton variation principle, the nonlinear wave equation of the finite deformation elastic rod can be obtained as follows [13-15]:

$$
\begin{gathered}
\frac{\partial^{2} U}{\partial t^{2}}-\frac{E}{\rho} \frac{\partial}{\partial x}\left(\frac{\partial U}{\partial x}\right) \\
=\frac{1}{2 \rho} \frac{\partial}{\partial x}\left[3 E\left(\frac{\partial U}{\partial x}\right)^{2}+E\left(\frac{\partial U}{\partial x}\right)^{3}+\rho \nu^{2} R^{2} \frac{\partial}{\partial x}\left(\frac{\partial^{2} U}{\partial t^{2}}\right)\right. \\
\left.-\mu \nu^{2} R^{2} \frac{\partial}{\partial x}\left(\frac{\partial^{2} U}{\partial x^{2}}\right)\right] .
\end{gathered}
$$

If we set $u=\partial U / \partial x,(1)$ may be rewritten as

$$
\begin{aligned}
\frac{\partial^{2} u}{\partial t^{2}} & -c_{0}^{2} \frac{\partial^{2} u}{\partial x^{2}} \\
& =\frac{\partial^{2}}{\partial x^{2}}\left[\frac{3 E}{2 \rho} u^{2}+\frac{E}{2 \rho} u^{3}+\frac{v^{2} R^{2}}{2}\left(\frac{\partial^{2} u}{\partial t^{2}}-v_{0}^{2} \frac{\partial^{2} u}{\partial x^{2}}\right)\right],
\end{aligned}
$$

where $c_{0}, v_{0}, \mu, \nu, E$, and $\rho$ are the linear elastic longitudinal wave velocity, the shear wave velocity, the shear modulus of material, Poisson ratio, the Young's modulus and the density of the rod, respectively. Equation (2) is the double nonlinear wave equation with respect to the axial displacement gradient finite deformation elastic rod. It is shown that when the longitudinal wave propagates, the shear wave also propagates as a result of the transverse Poisson effects.

We now outline the main steps of the extended mapping method. Consider a given nonlinear evolution equation:

$$
P\left(u, u_{t}, u_{x}, u_{t t}, u_{x x}, \ldots\right)=0
$$

We seek solutions of (3) by taking

$$
u(x, t)=u(\xi), \quad \xi=x-c t,
$$

where $c$ is a real constant. Under the transformation equation (4), equation (3) becomes an ordinary differential equation:

$$
Q\left(u, u^{\prime}, u^{\prime \prime}, \ldots\right)=0 .
$$

We assume that (5) has the solutions in the form

$$
u(\xi)=a_{0}+\sum_{i=1}^{m}\left[a_{i} f^{i}(\xi)+b_{i} f^{-i}(\xi)\right],
$$

where $a_{0}, a_{i}$, and $b_{i}(i=1,2, \ldots, m)$ are constants to be determined later. The integer $m$ in (6) can be determined by balancing the highest order nonlinear term and the highest order linear term of $u(\xi)$ in (5). The function $f(\xi)$ is the solution of the cubic nonlinear Klein-Gordon (NKG) equation

$$
f_{\xi \xi}=\lambda f+\delta f^{3}, \quad f_{\xi}^{2}=B_{0}+\lambda f^{2}+\left(\frac{\delta}{2}\right) f^{4},
$$

where $B_{0}, \lambda$, and $\delta$ are arbitrary constants. Substituting (6) and (7) into (5) results in an algebraic system of equations in powers of $f^{i}$ which will lead to the determination of parameters $a_{0}, a_{i}, b_{i}$, and $c$. Using (6) and the solutions of (7), we can obtain many explicit and exact traveling wave solutions of (3).

\section{Appling the Extended Mapping Method to (2)}

Substituting (4) into (2) yields

$$
\begin{gathered}
\left(c^{2}-c_{0}^{2}\right) \frac{d^{2} u}{d \xi^{2}}-\frac{d^{2}}{d \xi^{2}}\left(\frac{3 E}{2 \rho} u^{2}\right)-\frac{d^{2}}{d \xi^{2}}\left(\frac{E}{2 \rho} u^{3}\right) \\
-\frac{d^{2}}{d \xi^{2}}\left[\frac{v^{2} R^{2}}{2}\left(c^{2}-v_{0}^{2}\right) \frac{d^{2} u}{d \xi^{2}}\right]=0 .
\end{gathered}
$$

Integrating (8) with respect to $\xi$ twice and letting the integrating constant be zero, we have

$$
u^{\prime \prime}+\beta_{1} u+\beta_{2} u^{2}+\beta_{3} u^{3}=0
$$

where

$$
\begin{gathered}
\beta_{1}=-\frac{2\left(c^{2}-c_{0}^{2}\right)}{v^{2} R^{2}\left(c^{2}-v_{0}^{2}\right)}, \quad \beta_{2}=\frac{3 c_{0}^{2}}{v^{2} R^{2}\left(c^{2}-v_{0}^{2}\right)}, \\
\beta_{3}=\frac{c_{0}^{2}}{v^{2} R^{2}\left(c^{2}-v_{0}^{2}\right)} .
\end{gathered}
$$


Balancing of the highest derivative with nonlinear term in (9) gives $m=1$. The solution of (9) can be assumed as

$$
u(\xi)=a_{0}+a_{1} f(\xi)+b_{1} f^{-1}(\xi) .
$$

Substituting (7) and (11) into (9), we obtain

$$
\begin{aligned}
& 2 b_{1} B_{0}+b_{1}^{3} \beta_{3}+\left(b_{1}^{2} \beta_{2}+3 a_{0} b_{1}^{2} \beta_{3}\right) f \\
& +\left(b_{1} \beta_{1}+2 a_{0} b_{1} \beta_{2}+3 a_{0}^{2} b_{1} \beta_{3}+3 a_{1} b_{1}^{2} \beta_{3}+b_{1} \lambda\right) f^{2} \\
& +\left(a_{0} \beta_{1}+a_{0}^{2} \beta_{2}+2 a_{1} b_{1} \beta_{2}+a_{0}^{3} \beta_{3}+6 a_{0} a_{1} b_{1} \beta_{3}\right) f^{3} \\
& +\left(a_{1} \beta_{1}+2 a_{0} a_{1} \beta_{2}+3 a_{0}^{2} a_{1} \beta_{3}+3 a_{1}^{2} b_{1} \beta_{3}+a_{1} \lambda\right) f^{4} \\
& +\left(a_{1}^{2} \beta_{2}+3 a_{0} a_{1}^{2} \beta_{3}\right) f^{5}+\left(a_{1}^{3} \beta_{3}+a_{1} \delta\right) f^{6}=0 .
\end{aligned}
$$

Owing to $f$ being arbitrary solution of the cubic NKG equation, all the coefficients of different powers of $f$ in (12) should vanish. That means the following equations are established:

$$
\begin{gathered}
2 b_{1} B_{0}+b_{1}^{3} \beta_{3}=0, \\
b_{1}^{2} \beta_{2}+3 a_{0} b_{1}^{2} \beta_{3}=0, \\
b_{1} \beta_{1}+2 a_{0} b_{1} \beta_{2}+3 a_{0}^{2} b_{1} \beta_{3}+3 a_{1} b_{1}^{2} \beta_{3}+b_{1} \lambda=0, \\
a_{0} \beta_{1}+a_{0}^{2} \beta_{2}+2 a_{1} b_{1} \beta_{2}+a_{0}^{3} \beta_{3}+6 a_{0} a_{1} b_{1} \beta_{3}=0, \\
a_{1} \beta_{1}+2 a_{0} a_{1} \beta_{2}+3 a_{0}^{2} a_{1} \beta_{3}+3 a_{1}^{2} b_{1} \beta_{3}+a_{1} \lambda=0, \\
a_{1}^{2} \beta_{2}+3 a_{0} a_{1}^{2} \beta_{3}=0, \\
a_{1}^{3} \beta_{3}+a_{1} \delta=0 .
\end{gathered}
$$

These equations can easily be solved for the constants $a_{0}$, $a_{1}$, and $b_{1}$ in terms of parameters $\beta_{1}, \beta_{2}, \beta_{3}, B_{0}, \lambda$, and $\delta$. Solving (13) by Maple or Mathematica, we have the following.

The first set constants are

$$
a_{0}=-\frac{\beta_{2}}{3 \beta_{3}}, \quad a_{1}= \pm \sqrt{-\frac{\delta}{\beta_{3}}} .
$$

The second set constants are

$$
a_{0}=-\frac{\beta_{2}}{3 \beta_{3}}, \quad b_{1}= \pm \sqrt{-\frac{2 B_{0}}{\beta_{3}}} .
$$

The third set constants are

$a_{0}=-\frac{3 \beta_{1}}{2 \beta_{2}}, \quad a_{1}= \pm \sqrt{-\frac{\delta}{\beta_{3}}}, \quad b_{1}= \pm \frac{\beta_{1}-2 \lambda}{6 \sqrt{-\delta \beta_{3}}}$.

Hence solutions of (8) turn out to be

$$
\begin{gathered}
u(\xi)=-\frac{\beta_{2}}{3 \beta_{3}} \pm \sqrt{-\frac{\delta}{\beta_{3}}} f(\xi), \\
u(\xi)=-\frac{\beta_{2}}{3 \beta_{3}} \pm \sqrt{-\frac{2 B_{0}}{\beta_{3}}} f(\xi)^{-1}, \\
u(\xi)=-\frac{3 \beta_{1}}{2 \beta_{2}} \pm \sqrt{-\frac{\delta}{\beta_{3}}} f(\xi) \pm \frac{\beta_{1}-2 \lambda}{6 \sqrt{-\delta \beta_{3}}} f(\xi)^{-1} .
\end{gathered}
$$

From the general solutions of the cubic NKG equation (7), we have four types of traveling wave solutions of (2).

\subsection{Trigonometric Function Solutions}

Case 1. As $\lambda=2, \delta=2, B_{0}=1$, and $f(\xi)=\tan (\xi)$,

$$
\begin{gathered}
u(\xi)=-\frac{\beta_{2}}{3 \beta_{3}} \pm \sqrt{-\frac{2}{\beta_{3}}} \tan (\xi), \\
u(\xi)=-\frac{\beta_{2}}{3 \beta_{3}} \pm \sqrt{-\frac{2}{\beta_{3}}} \cot (\xi), \\
u(\xi)=-\frac{3 \beta_{1}}{2 \beta_{2}} \pm \sqrt{-\frac{2}{\beta_{3}}} \tan (\xi) \pm \frac{\beta_{1}-4}{6 \sqrt{-2 \beta_{3}}} \cot (\xi) .
\end{gathered}
$$

Case 2. As $\lambda=-1, \delta=2, B_{0}=0$, and $f(\xi)=\sec (\xi)$,

$$
\begin{gathered}
u(\xi)=-\frac{\beta_{2}}{3 \beta_{3}} \pm \sqrt{-\frac{2}{\beta_{3}}} \sec (\xi), \\
u(\xi)=-\frac{3 \beta_{1}}{2 \beta_{2}} \pm \sqrt{-\frac{2}{\beta_{3}}} \sec (\xi) \pm \frac{\beta_{1}+2}{6 \sqrt{-2 \beta_{3}}} \cos (\xi) .
\end{gathered}
$$

Case 3. As $\lambda=2, \delta=2, B_{0}=1$, and $f(\xi)=\cot (\xi)$,

$$
\begin{gathered}
u(\xi)=-\frac{\beta_{2}}{3 \beta_{3}} \pm \sqrt{-\frac{2}{\beta_{3}}} \cot (\xi), \\
u(\xi)=-\frac{\beta_{2}}{3 \beta_{3}} \pm \sqrt{-\frac{2}{\beta_{3}}} \tan (\xi), \\
u(\xi)=-\frac{3 \beta_{1}}{2 \beta_{2}} \pm \sqrt{-\frac{2}{\beta_{3}}} \cot (\xi) \pm \frac{\beta_{1}-4}{6 \sqrt{-2 \beta_{3}}} \tan (\xi) .
\end{gathered}
$$

Case 4. As $\lambda=-1, \delta=2, B_{0}=0$, and $f(\xi)=\csc (\xi)$,

$$
\begin{gathered}
u(\xi)=-\frac{\beta_{2}}{3 \beta_{3}} \pm \sqrt{-\frac{2}{\beta_{3}}} \csc (\xi), \\
u(\xi)=-\frac{3 \beta_{1}}{2 \beta_{2}} \pm \sqrt{-\frac{2}{\beta_{3}}} \csc (\xi) \pm \frac{\beta_{1}+2}{6 \sqrt{-2 \beta_{3}}} \sin (\xi) .
\end{gathered}
$$

Equations (18)-(21) are trigonometric function periodic wave solutions of (2). 


\subsection{Solitary Wave Solutions}

Case 1. We know that when $\lambda=-2, \delta=2$, and $B_{0}=1$, the solution of (7) reads $f(\xi)=\tanh (\xi)$. Thus, from (17), the corresponding solutions of (2) read

$$
\begin{gathered}
u(\xi)=-\frac{\beta_{2}}{3 \beta_{3}} \pm \sqrt{-\frac{2}{\beta_{3}}} \tanh (\xi), \\
u(\xi)=-\frac{\beta_{2}}{3 \beta_{3}} \pm \sqrt{-\frac{2}{\beta_{3}}} \operatorname{coth}(\xi), \\
u(\xi)=-\frac{3 \beta_{1}}{2 \beta_{2}} \pm \sqrt{-\frac{2}{\beta_{3}}} \tanh (\xi) \pm \frac{\beta_{1}+4}{6 \sqrt{-2 \beta_{3}}} \operatorname{coth}(\xi),
\end{gathered}
$$

where $\tanh (\xi)$ and $\operatorname{coth}(\xi)$ are hyperbolic functions.

Case 2. As $\lambda=1, \delta=-2, B_{0}=0$, and $f(\xi)=\operatorname{sech}(\xi)$,

$$
\begin{gathered}
u(\xi)=-\frac{\beta_{2}}{3 \beta_{3}} \pm \sqrt{\frac{2}{\beta_{3}}} \operatorname{sech}(\xi), \\
u(\xi)=-\frac{3 \beta_{1}}{2 \beta_{2}} \pm \sqrt{\frac{2}{\beta_{3}}} \operatorname{sech}(\xi) \pm \frac{\beta_{1}-2}{6 \sqrt{2 \beta_{3}}} \cosh (\xi),
\end{gathered}
$$

where $\operatorname{sech}(\xi)$ and $\cosh (\xi)$ are hyperbolic functions.

Case 3. As $\lambda=1, \delta=2, B_{0}=0$, and $f(\xi)=\operatorname{csch}(\xi)$,

$$
\begin{gathered}
u(\xi)=-\frac{\beta_{2}}{3 \beta_{3}} \pm \sqrt{-\frac{2}{\beta_{3}}} \operatorname{csch}(\xi), \\
u(\xi)=-\frac{3 \beta_{1}}{2 \beta_{2}} \pm \sqrt{-\frac{2}{\beta_{3}}} \operatorname{csch}(\xi) \pm \frac{\beta_{1}-2}{6 \sqrt{-2 \beta_{3}}} \sinh (\xi),
\end{gathered}
$$

where $\operatorname{csch}(\xi)$ and $\sinh (\xi)$ are hyperbolic functions.

Case 4. As $\lambda=-2, \delta=2, B_{0}=1$, and $f(\xi)=\operatorname{coth}(\xi)$,

$$
\begin{gathered}
u(\xi)=-\frac{\beta_{2}}{3 \beta_{3}} \pm \sqrt{-\frac{2}{\beta_{3}}} \operatorname{coth}(\xi), \\
u(\xi)=-\frac{\beta_{2}}{3 \beta_{3}} \pm \sqrt{-\frac{2}{\beta_{3}}} \tanh (\xi), \\
u(\xi)=-\frac{3 \beta_{1}}{2 \beta_{2}} \pm \sqrt{-\frac{2}{\beta_{3}}} \operatorname{coth}(\xi) \pm \frac{\beta_{1}+4}{6 \sqrt{-2 \beta_{3}}} \tanh (\xi) .
\end{gathered}
$$

The solutions in the form $\tanh (\xi), \operatorname{sech}(\xi)$, and $\operatorname{csch}(\xi)$ are kink solutions, soliton solutions, and diverging solutions, respectively.

\subsection{Jacobian Elliptic Function Solutions}

Case 1. As $\lambda=-\left(1+k^{2}\right), \delta=2 k^{2}, B_{0}=1$, and $f(\xi)=\operatorname{sn}(\xi)$,

$$
\begin{gathered}
u(\xi)=-\frac{\beta_{2}}{3 \beta_{3}} \pm \sqrt{-\frac{2 k^{2}}{\beta_{3}}} \operatorname{sn}(\xi), \\
u(\xi)=-\frac{\beta_{2}}{3 \beta_{3}} \pm \sqrt{-\frac{2}{\beta_{3}}}\left(\frac{1}{\operatorname{sn}(\xi)}\right), \\
u(\xi)=-\frac{3 \beta_{1}}{2 \beta_{2}} \pm \sqrt{-\frac{2 k^{2}}{\beta_{3}}} \operatorname{sn}(\xi) \pm \frac{\beta_{1}+2\left(1+k^{2}\right)}{6 \sqrt{-2 k^{2} \beta_{3}} \operatorname{sn}(\xi)}
\end{gathered}
$$

where $\operatorname{sn}(\xi)$ is Jacobian elliptic function with modulus $k$.

Case 2. As $\lambda=2 k^{2}-1, \delta=-2 k^{2}, B_{0}=1-k^{2}$, and $f(\xi)=\mathrm{cn}(\xi)$,

$$
\begin{gathered}
u(\xi)=-\frac{\beta_{2}}{3 \beta_{3}} \pm \sqrt{\frac{2 k^{2}}{\beta_{3}}} \mathrm{cn}(\xi), \\
u(\xi)=-\frac{\beta_{2}}{3 \beta_{3}} \pm \sqrt{-\frac{2\left(1-k^{2}\right)}{\beta_{3}}}\left(\frac{1}{\mathrm{cn}(\xi)}\right), \\
u(\xi)=-\frac{3 \beta_{1}}{2 \beta_{2}} \pm \sqrt{\frac{2 k^{2}}{\beta_{3}}} \operatorname{cn}(\xi) \pm \frac{\beta_{1}-2\left(2 k^{2}-1\right)}{6 \sqrt{2 k^{2} \beta_{3}} \operatorname{cn}(\xi)},
\end{gathered}
$$

where $\mathrm{cn}(\xi)$ is Jacobian elliptic function with modulus $k$.

Case 3. As $\lambda=2-k^{2}, \delta=-2, B_{0}=-1+k^{2}$, and $f(\xi)=\operatorname{dn}(\xi)$,

$$
\begin{aligned}
& u(\xi)=-\frac{\beta_{2}}{3 \beta_{3}} \pm \sqrt{\frac{2}{\beta_{3}}} \operatorname{dn}(\xi), \\
& u(\xi)=-\frac{\beta_{2}}{3 \beta_{3}} \pm \sqrt{-\frac{2\left(-1+k^{2}\right)}{\beta_{3}}}\left(\frac{1}{\operatorname{dn}(\xi)}\right), \\
& u(\xi)=-\frac{3 \beta_{1}}{2 \beta_{2}} \pm \sqrt{\frac{2}{\beta_{3}}} \operatorname{dn}(\xi) \pm \frac{\beta_{1}-2\left(2-k^{2}\right)}{6 \sqrt{2 \beta_{3}} \operatorname{dn}(\xi)},
\end{aligned}
$$

where $\operatorname{dn}(\xi)$ is Jacobian elliptic function with modulus $k$.

Case 4. As $\lambda=2-k^{2}, \delta=2\left(1-k^{2}\right), B_{0}=1$, and $f(\xi)=\operatorname{sc}(\xi)$,

$$
\begin{gathered}
u(\xi)=-\frac{\beta_{2}}{3 \beta_{3}} \pm \sqrt{-\frac{2\left(1-k^{2}\right)}{\beta_{3}}} \mathrm{sc}(\xi), \\
u(\xi)=-\frac{\beta_{2}}{3 \beta_{3}} \pm \sqrt{-\frac{2}{\beta_{3}}\left(\frac{1}{\operatorname{sc}(\xi)}\right),} \\
u(\xi)=-\frac{3 \beta_{1}}{2 \beta_{2}} \pm \sqrt{-\frac{2\left(1-k^{2}\right)}{\beta_{3}}} \mathrm{sc}(\xi) \\
\pm \frac{\beta_{1}-2\left(2-k^{2}\right)}{6 \sqrt{-2\left(1-k^{2}\right) \beta_{3}} \operatorname{sc}(\xi)}
\end{gathered}
$$

where $\operatorname{sc}(\xi)=\operatorname{sn}(\xi) / \operatorname{cn}(\xi)$ is Jacobian elliptic function with modulus $k$. 
Case 5. As $\lambda=2 k^{2}-1, \delta=2 k^{2}\left(k^{2}-1\right), B_{0}=1$, and $f(\xi)=$ $\operatorname{sd}(\xi)$,

$$
\begin{gathered}
u(\xi)=-\frac{\beta_{2}}{3 \beta_{3}} \pm \sqrt{-\frac{2 k^{2}\left(k^{2}-1\right)}{\beta_{3}}} \operatorname{sd}(\xi), \\
u(\xi)=-\frac{\beta_{2}}{3 \beta_{3}} \pm \sqrt{-\frac{2}{\beta_{3}}\left(\frac{1}{\operatorname{sd}(\xi)}\right),} \\
u(\xi)=-\frac{3 \beta_{1}}{2 \beta_{2}} \pm \sqrt{-\frac{2 k^{2}\left(k^{2}-1\right)}{\beta_{3}}} \operatorname{sd}(\xi) \\
\pm \frac{\beta_{1}-2\left(2 k^{2}-1\right)}{6 \sqrt{-2 k^{2}\left(k^{2}-1\right) \beta_{3}} \operatorname{sd}(\xi)},
\end{gathered}
$$

where $\operatorname{sd}(\xi)=\operatorname{sn}(\xi) / \operatorname{dn}(\xi)$ is Jacobian elliptic function with modulus $k$.

Case 6. As $\lambda=-\left(\left(1+k^{2}\right) /(1+k)^{2}\right), \delta=2 k^{2} /(k+1)^{2}, B_{0}=$ $1 /(1+k)^{2}$, and $f(\xi)=\operatorname{cd}(\xi)$,

$$
\begin{gathered}
u(\xi)=-\frac{\beta_{2}}{3 \beta_{3}} \pm \sqrt{-\frac{2 k^{2}}{\beta_{3}(k+1)^{2}}} \mathrm{~cd}(\xi), \\
u(\xi)=-\frac{\beta_{2}}{3 \beta_{3}} \pm \sqrt{-\frac{2}{\beta_{3}(1+k)^{2}}}\left(\frac{1}{\mathrm{~cd}(\xi)}\right), \\
u(\xi)=-\frac{3 \beta_{1}}{2 \beta_{2}} \pm \sqrt{-\frac{2 k^{2}}{\beta_{3}(k+1)^{2}}} \mathrm{~cd}(\xi) \\
\quad \pm \frac{\beta_{1}+2\left(\left(1+k^{2}\right) /(1+k)^{2}\right)}{6 \sqrt{-\left(2 k^{2} /(k+1)^{2}\right) \beta_{3}} \mathrm{~cd}(\xi)},
\end{gathered}
$$

where $\operatorname{cd}(\xi)=\operatorname{cn}(\xi) / \mathrm{dn}(\xi)$ is Jacobian elliptic function with modulus $k$.

Case 7. As $\lambda=2-k^{2}, \delta=2, B_{0}=1-k^{2}$, and $f(\xi)=\operatorname{cs}(\xi)$,

$$
\begin{gathered}
u(\xi)=-\frac{\beta_{2}}{3 \beta_{3}} \pm \sqrt{-\frac{2}{\beta_{3}}} \operatorname{cs}(\xi), \\
u(\xi)=-\frac{\beta_{2}}{3 \beta_{3}} \pm \sqrt{-\frac{2\left(1-k^{2}\right)}{\beta_{3}}}\left(\frac{1}{\operatorname{cs}(\xi)}\right), \\
u(\xi)=-\frac{3 \beta_{1}}{2 \beta_{2}} \pm \sqrt{-\frac{2}{\beta_{3}}} \operatorname{cs}(\xi) \pm \frac{\beta_{1}-2\left(2-k^{2}\right)}{6 \sqrt{-2 \beta_{3}} \operatorname{cs}(\xi)},
\end{gathered}
$$

where $\operatorname{cs}(\xi)=\operatorname{cn}(\xi) / \operatorname{sn}(\xi)$ is Jacobian elliptic function with modulus $k$.
Case 8. As $\lambda=2 k^{2}-1, \delta=2, B_{0}=k^{4}-k^{2}$, and $f(\xi)=\mathrm{ds}(\xi)$,

$$
\begin{gathered}
u(\xi)=-\frac{\beta_{2}}{3 \beta_{3}} \pm \sqrt{-\frac{2}{\beta_{3}}} \mathrm{ds}(\xi), \\
u(\xi)=-\frac{\beta_{2}}{3 \beta_{3}} \pm \sqrt{-\frac{2\left(k^{4}-k^{2}\right)}{\beta_{3}}}\left(\frac{1}{\mathrm{ds}(\xi)}\right), \\
u(\xi)=-\frac{3 \beta_{1}}{2 \beta_{2}} \pm \sqrt{-\frac{2}{\beta_{3}}} \mathrm{ds}(\xi) \pm \frac{\beta_{1}-2\left(2 k^{2}-1\right)}{6 \sqrt{-2 \beta_{3}} \mathrm{ds}(\xi)},
\end{gathered}
$$

where $\mathrm{ds}(\xi)=\operatorname{dn}(\xi) / \operatorname{sn}(\xi)$ is Jacobian elliptic function with modulus $k$.

Case 9. As $\lambda=-k^{2}, \delta=2, B_{0}=-1+k^{2}$, and $f(\xi)=\mathrm{dc}(\xi)$,

$$
u(\xi)=-\frac{\beta_{2}}{3 \beta_{3}} \pm \sqrt{-\frac{2}{\beta_{3}}} \mathrm{dc}(\xi),
$$

$$
\begin{gathered}
u(\xi)=-\frac{\beta_{2}}{3 \beta_{3}} \pm \sqrt{-\frac{2\left(-1+k^{2}\right)}{\beta_{3}}}\left(\frac{1}{\mathrm{dc}(\xi)}\right), \\
u(\xi)=-\frac{3 \beta_{1}}{2 \beta_{2}} \pm \sqrt{-\frac{2}{\beta_{3}}} \mathrm{dc}(\xi) \pm \frac{\beta_{1}+2 k^{2}}{6 \sqrt{-2 \beta_{3}} \mathrm{dc}(\xi)},
\end{gathered}
$$

where $\operatorname{dc}(\xi)=\operatorname{dn}(\xi) / \operatorname{cn}(\xi)$ is Jacobian elliptic function with modulus $k$.

In addition when $k \rightarrow 1$, the functions $\operatorname{sn}(\xi), \mathrm{cn}(\xi)$, and $\operatorname{dn}(\xi)$ degenerate as $\tanh (\xi), \operatorname{sech}(\xi)$, and $\operatorname{sech}(\xi)$, respectively, while when $k \rightarrow 0, \operatorname{sn}(\xi), \operatorname{cn}(\xi)$, and $\operatorname{dn}(\xi)$ degenerate as $\sin (\xi), \cos (\xi)$, and 1 , respectively. We can obtain hyperbolic function solutions and trigonometric function solutions in the limit cases as $k \rightarrow 1$ and $k \rightarrow 0$.

3.4. Weierstrass Elliptic Function Solutions. We get the following Weierstrass elliptic function solutions from (17).

Case 1. As $g_{2}=\left(4 \lambda^{2}-6 B_{0} \delta\right) / 3, g_{3}=4 \lambda\left(-2 \lambda^{2}+(9 / 2) B_{0} \delta\right) / 27$, and $f(\xi)=\sqrt{(2 / \delta)\left(\wp\left(\xi ; g_{2}, g_{3}\right)-(\lambda / 3)\right)}$,

$$
\begin{array}{r}
u(\xi)=-\frac{\beta_{2}}{3 \beta_{3}} \pm \sqrt{-\frac{2}{\beta_{3}}\left(\wp\left(\xi ; g_{2}, g_{3}\right)-\frac{\lambda}{3}\right)}, \\
u(\xi)=-\frac{\beta_{2}}{3 \beta_{3}} \pm \sqrt{-\frac{4 B_{0}}{\beta_{3} \delta\left(\wp\left(\xi ; g_{2}, g_{3}\right)-(\lambda / 3)\right)}}, \\
u(\xi)=-\frac{3 \beta_{1}}{2 \beta_{2}} \pm \sqrt{-\frac{2}{\beta_{3}\left(\wp\left(\xi ; g_{2}, g_{3}\right)-\frac{\lambda}{3}\right)}}, \\
\pm \frac{\beta_{1}-2 \lambda}{6 \sqrt{-\delta \beta_{3}}} \sqrt{\frac{\delta}{2\left(\wp\left(\xi ; g_{2}, g_{3}\right)-(\lambda / 3)\right)}},
\end{array}
$$

where $\wp\left(\xi ; g_{2}, g_{3}\right)$ is Weierstrass elliptic function satisfying

$$
\wp^{\prime 2}(\xi)=4 \wp^{3}(\xi)-g_{2} \wp(\xi)-g_{3} .
$$


Case 2. As $g_{2}=\left(4 \lambda^{2}-6 B_{0} \delta\right) / 3, g_{3}=4 \lambda\left(-2 \lambda^{2}+(9 / 2) B_{0} \delta\right) / 27$, and $f(\xi)=\sqrt{3 B_{0} /\left(3 \wp\left(\xi ; g_{2}, g_{3}\right)-\lambda\right)}$,

$$
\begin{aligned}
u(\xi)= & -\frac{\beta_{2}}{3 \beta_{3}} \pm \sqrt{-\frac{3 B_{0} \delta}{\beta_{3}\left(3 \wp\left(\xi ; g_{2}, g_{3}\right)-\lambda\right)}}, \\
u(\xi)= & -\frac{\beta_{2}}{3 \beta_{3}} \pm \sqrt{-\frac{2\left(3 \wp\left(\xi ; g_{2}, g_{3}\right)-\lambda\right)}{3 \beta_{3}}}, \\
u(\xi)= & -\frac{3 \beta_{1}}{2 \beta_{2}} \pm \sqrt{-\frac{3 B_{0} \delta}{\beta_{3}\left(3 \wp\left(\xi ; g_{2}, g_{3}\right)-\lambda\right)}} \\
& \pm \frac{\beta_{1}-2 \lambda}{6 \sqrt{-\delta \beta_{3}}} \sqrt{\frac{3 \wp\left(\xi ; g_{2}, g_{3}\right)-\lambda}{3 B_{0}}} .
\end{aligned}
$$

In particular, the solution of (45) can be written in terms of Jacobian elliptic function as

$$
\wp\left(\xi ; g_{2}, g_{3}\right)=e_{2}-\left(e_{2}-e_{3}\right) \mathrm{cn}^{2}\left(\sqrt{e_{1}-e_{3}} \xi\right),
$$

where $e_{i}\left(i=1,2,3 ; e_{1} \geq e_{2} \geq e_{3}\right)$ are three toots of the cubic equation $4 z^{3}-g_{2} z-g_{3}=0$. We rewrite (42) as the more familiar form in terms of Jacobian elliptic function

$u(\xi)$

$$
=-\frac{\beta_{2}}{3 \beta_{3}} \pm \sqrt{-\frac{2}{\beta_{3}}\left(\left[e_{2}-\left(e_{2}-e_{3}\right) \mathrm{cn}^{2}\left(\sqrt{e_{1}-e_{3}} \xi\right)\right]-\frac{\lambda}{3}\right)} .
$$

Because $k \rightarrow 1$ and $\operatorname{cn}(\xi) \rightarrow \operatorname{sech}(\xi),(48)$ can be written as the form

$$
\begin{aligned}
& u(\xi) \\
& =-\frac{\beta_{2}}{3 \beta_{3}} \pm \sqrt{-\frac{2}{\beta_{3}}\left(\left[e_{2}-\left(e_{2}-e_{3}\right) \operatorname{sech}^{2}\left(\sqrt{e_{1}-e_{3}} \xi\right)\right]-\frac{\lambda}{3}\right)} .
\end{aligned}
$$

Similarly, we can write (43)-(46) as other forms in terms of Jacobian elliptic function or hyperbolic function.

Remark 1. By using (17) and letting $B_{0}, \lambda$, and $\delta$ equal to the corresponding value, more traveling wave solutions can be obtained. We omit them in this paper.

Remark 2. Among them, (22), (25), (29), and (33) have been obtained by Liu and Zhang [13] using the Jacobi elliptic function expansion method. To our knowledge, other solutions seem not to be reported before. Our new results ensure that the extended mapping method not only recovers all of the solutions reported in [13] but also discovers many new ones.

\section{Conclusions}

We present a number of novel solutions of the nonlinear wave equation of an elastic rod under finite deformation. To study these solutions further so as to investigate the stability and physical significance of these solutions is worthwhile. It is also important to find the explanations of certain physical phenomena related to the elastic rod. The results show that the proposed method is reliable and effective and gives more solutions. This method can be also applied to other kinds of NLEEs.

Equation (2) includes both the finite deformation and the lateral Poisson effect. Because the lateral inertia and lateral shear are introduced simultaneously, the rod has two kinds of geometric dispersions. Owing to the interaction and constraint between nonlinearity and dispersion or dissipation effects, steady traveling wave may exist in the rod. Equations (18)-(49) indicate that the characteristics of these solutions are related to $\beta_{3}$. For example, when $\beta_{3}<0$, kink solution exists in Section 3.2, and when $\beta_{3}>0$, soliton solution and diverging solution exist in Section 3.2. The similar analysis can be applied to find other solutions.

\section{Conflict of Interests}

The authors declare that there is no conflict of interests regarding the publication of this paper.

\section{Acknowledgments}

The authors would like to express their sincere thanks to editors and referees for their valuable suggestions and comments. This work was supported by the National Natural Science Foundation of China under Grant no. 11164013, the Scientific Research Foundation of the Higher Education Institutions of Gansu Province under Grant no. 2014A-053, and the Young Scholars Science Foundation of Lanzhou Jiaotong University under Grant no. 2013026.

\section{References}

[1] G. B. Whitham, Linear and Nonlinear Waves, John Wiley \& Sons, New York, NY, USA, 1974.

[2] P. L. Bhatnagar, Nonlinear Waves in One-Dimensional Dispersive Systems, Clarendon Press, Oxford, UK, 1st edition, 1979.

[3] C. S. Gardner, J. M. Greene, M. D. Kruskal, and R. M. Miura, "Method for solving the Korteweg-de Vries equation," Physical Review Letters, vol. 19, no. 19, pp. 1095-1097, 1967.

[4] R. Hirota and J. Satsuma, "Soliton solutions of a coupled Korteweg-de Vries equation," Physics Letters A, vol. 85, no. 8-9, pp. 407-408, 1981.

[5] E. J. Parkes and B. R. Duffy, "An automated tanh-function method for finding solitary wave solutions to non-linear evolution equations," Computer Physics Communications, vol. 98, no. 3, pp. 288-300, 1996.

[6] E. G. Fan, "Extended tanh-function method and its applications to nonlinear equations," Physics Letters A, vol. 277, no. 4-5, pp. 212-218, 2000.

[7] A. Bekir, "New solitons and periodic wave solutions for some nonlinear physical models by using the sine-cosine method," Physica Scripta, vol. 77, no. 4, Article ID 045008, 2008.

[8] M. Wang, "Exact solutions for a compound KdV-Burgers equation," Physics Letters A, vol. 213, no. 5-6, pp. 279-287, 1996. 
[9] M. A. Abdou, "The extended F-expansion method and its application for a class of nonlinear evolution equations," Chaos, Solitons \& Fractals, vol. 31, no. 1, pp. 95-104, 2007.

[10] J.-H. He and X.-H. Wu, "Exp-function method for nonlinear wave equations," Chaos, Solitons and Fractals, vol. 30, no. 3, pp. 700-708, 2006.

[11] S. Y. Lou and G. J. Ni, "The relations among a special type of solutions in some $(D+1)$-dimensional nonlinear equations," Journal of Mathematical Physics, vol. 30, no. 7, pp. 1614-1620, 1989.

[12] C. Bai, H. Zhao, H. Xu, and X. Zhang, "New traveling wave solutions for a class of nonlinear evolution equations," International Journal of Modern Physics B: Condensed Matter Physics: Statistical Physics: Applied Physics, vol. 25, no. 2, pp. 319-327, 2011.

[13] Z. F. Liu and S. Y. Zhang, "Nonlinear waves and periodic solution in finite deformation elastic rod," Acta Mechanica Solida Sinica, vol. 19, no. 1, pp. 1-8, 2006.

[14] Z. Liu, T. Wang, and S. Zhang, "Study on propagation of nonlinear flexural waves in the beams," Chinese Journal of Theoretical and Applied Mechanics, vol. 39, no. 2, pp. 238-244, 2007.

[15] S.-Y. Zhang and Z.-F. Liu, "Three kinds of nonlinear dispersive waves in elastic rods with finite deformation," Applied Mathematics and Mechanics, vol. 29, no. 7, pp. 909-917, 2008. 


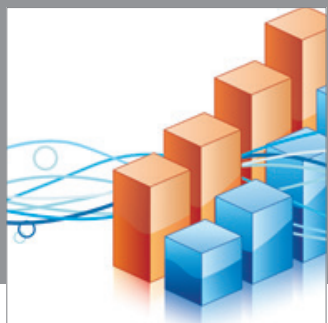

Advances in

Operations Research

mansans

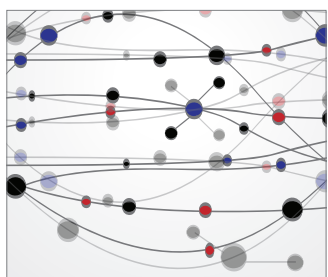

The Scientific World Journal
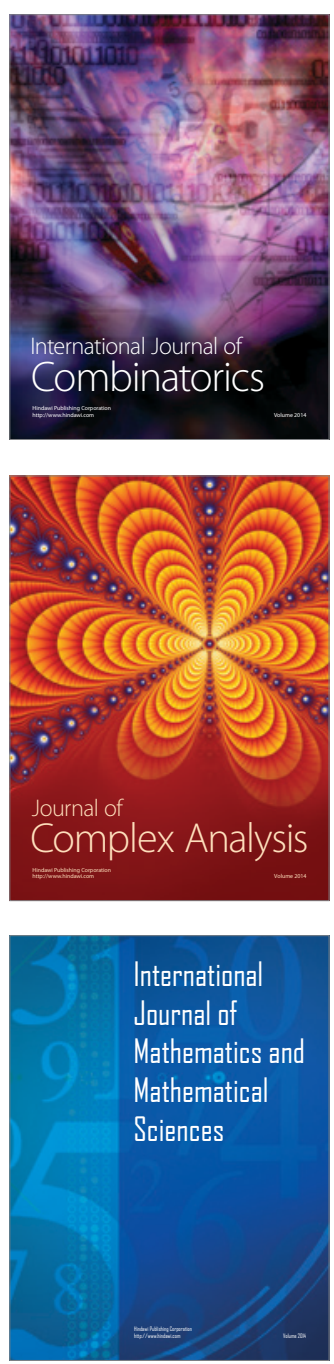
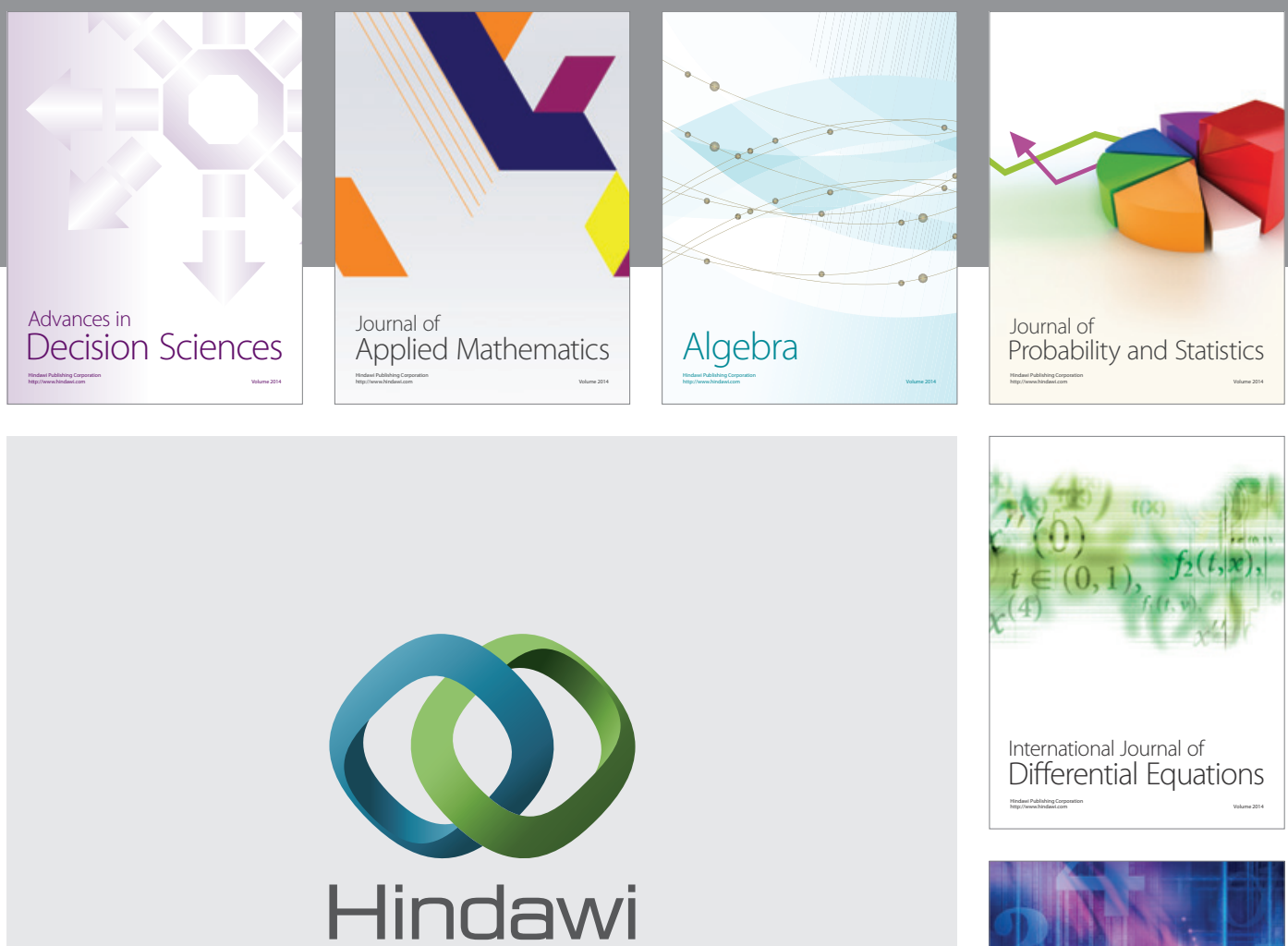

Submit your manuscripts at http://www.hindawi.com
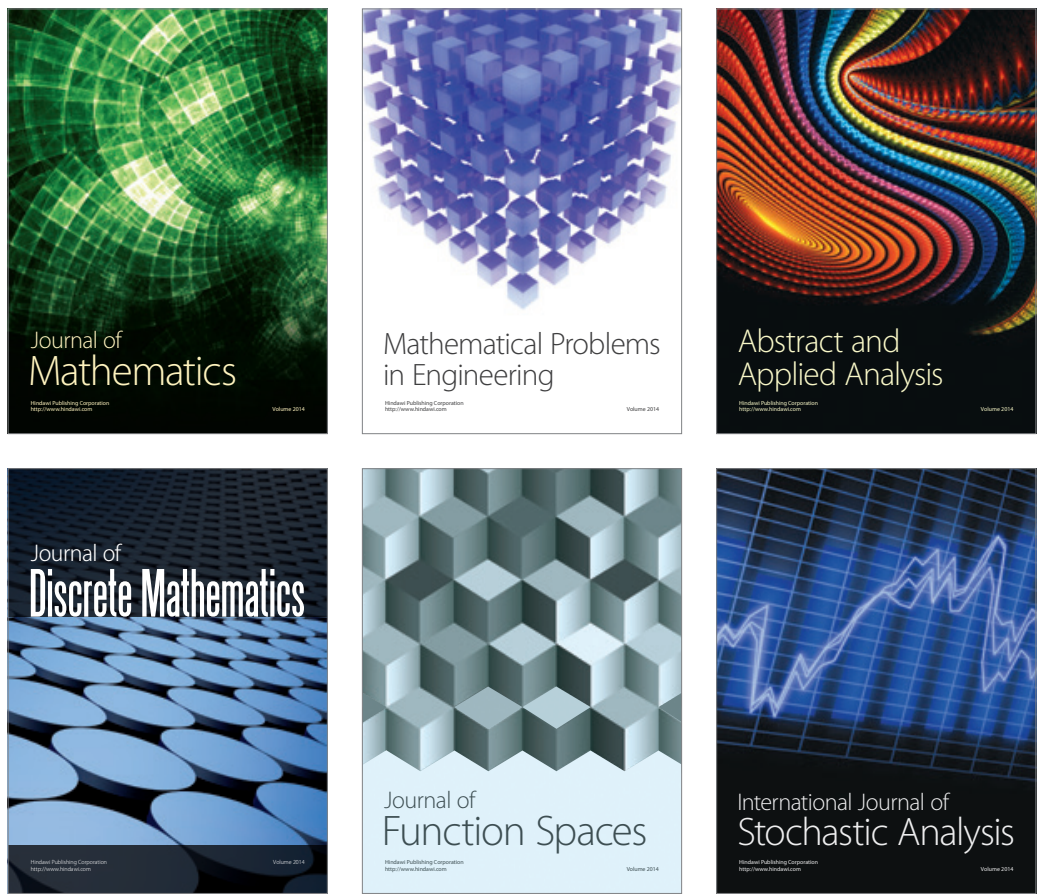

Journal of

Function Spaces

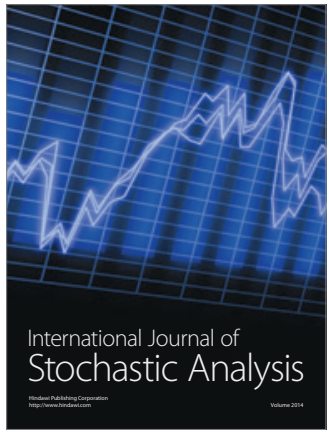

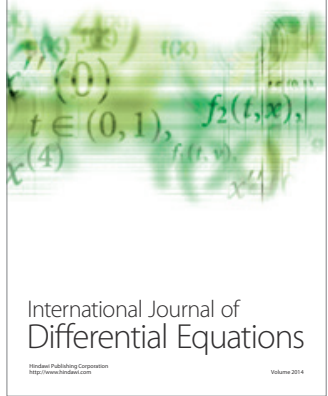
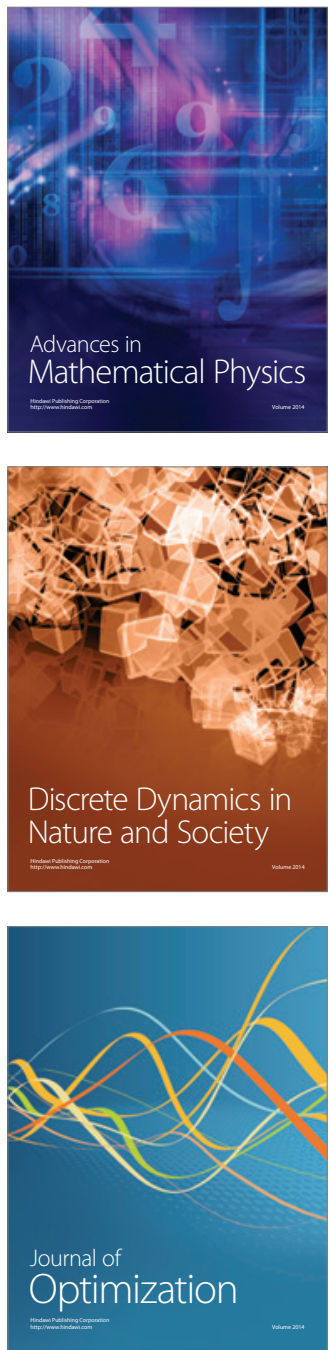